\title{
Partição de nicho entre espécies da família Leptodactylidae: um breve relato sobre
}

\section{irmãos}

\author{
Niche partitioning between species of the Leptodactylidae family: a brief account of siblings \\ División de nicho entre especies de la familia Leptodactylidae: una breve descripción de los
}

hermanos

Recebido: 10/02/2022 | Revisado: 17/02/2022 | Aceito: 18/02/2022 | Publicado: 26/02/2022

\author{
Wenner Justino Bezerra de Brito \\ ORCID: https://orcid.org/0000-0003-4582-7207 \\ Universidade Federal da Paraíba, Brasil \\ E-mail: wennerbrito10@gmail.com \\ Rafael Dioni Leandro Costa \\ ORCID: https://orcid.org/0000-0002-2118-4561 \\ Universidade Federal da Paraíba, Brasil \\ E-mail: rafaeldioni2011@hotmail.com
}

\begin{abstract}
Resumo
Trabalhos sobre partição de recursos entre espécies de anfíbios filogeneticamente relacionados são escassos para o domínio da Caatinga, sendo a maior parte dos estudos desenvolvidos para região de Mata Atlântica. Portanto, objetivouse avaliar o padrão de distribuição espacial e temporal de 9 espécies da família Leptodactylidae na região de Caatinga do Estado de Pernambuco. Foram realizadas observações por via do método de Procura Visual Limitada por Tempo (PVLT) durante 18 meses nas imediações da propriedade rural Cachoeira de Maria Mendes no município de Tabira. Ocorreram 594 avistamentos de indivíduos, pertencentes a 9 espécies integrantes da família Leptodactylidae. Foram feitas análises para evidenciar a sobreposição do uso do habitat e relacionar a riqueza versus variáveis abióticas. Os resultados sugeriram máxima sobreposição do uso de habitat, entretanto, a menor sobreposição foi evidenciada para as espécies Leptodactylus troglodytes versus Leptodactylus fuscus que integram o grupo fuscus da família Leptodactylidae. Além disso, todas as espécies amostradas apresentaram comportamento generalista quanto ao uso de habitat, com uma maior ocorrência em ambientes antrópicos.
\end{abstract}

Palavras-chave: Anfíbios; Distribuição; Generalista; Sazonalidade; Semiárido.

\begin{abstract}
Studies on the partition of resources between phylogenetically related amphibian species are scarce for the Caatinga domain, with most studies developed for the Atlantic Forest region. Therefore, the objective was to evaluate the spatial and temporal distribution pattern of 9 species of the Leptodactylidae family in the Caatinga region of the State of Pernambuco. Observations were carried out using the Time Limited Visual Search (PVLT) method for 18 months in the vicinity of the Cachoeira de Maria Mendes rural property in the municipality of Tabira. There were 594 sightings of individuals belonging to 9 species belonging to the Leptodactylidae family. Analyzes were carried out to evidence the overlap of habitat use and to relate richness versus abiotic variables. The results suggested maximum overlap of habitat use, however, the smallest overlap was evidenced for the species Leptodactylus troglodytes versus Leptodactylus fuscus that integrate the fuscus group of the Leptodactylidae family. In addition, all species sampled showed generalist behavior regarding habitat use, with a higher occurrence in anthropic environments.
\end{abstract}

Keywords: Amphibians; Distribution; Generalist; Seasonality; Semiárido.

\section{Resumen}

Los estudios sobre la partición de recursos entre especies de anfibios filogenéticamente relacionadas son escasos para el dominio Caatinga, con la mayoría de los estudios desarrollados para la región del Bosque Atlántico. Por lo tanto, el objetivo fue evaluar el patrón de distribución espacial y temporal de 9 especies de la familia Leptodactylidae en la región Caatinga del Estado de Pernambuco. Las observaciones se realizaron mediante el método de Búsqueda Visual por Tiempo Limitado (PVLT) durante 18 meses en las inmediaciones de la propiedad rural Cachoeira de Maria Mendes en el municipio de Tabira. Hubo 594 avistamientos de individuos pertenecientes a 9 especies pertenecientes a la familia Leptodactylidae. Se realizaron análisis para evidenciar la superposición de uso de hábitat y relacionar riqueza versus variables abióticas. Los resultados sugirieron máxima superposición de uso de hábitat, sin embargo, la menor superposición se evidenció para la especie Leptodactylus troglodytes versus Leptodactylus fuscus que integran el grupo fuscus de la familia Leptodactylidae. Además, todas las especies muestreadas mostraron un comportamiento generalista en cuanto al uso del hábitat, con una mayor ocurrencia en ambientes antrópicos.

Palabras clave: Anfibios; Distribución; Generalista; Estacionalidad; Semiárido. 


\section{Introdução}

O conhecimento sobre distribuição temporal e espacial de organismos é essencial para o entendimento de diversos processos ecológicos relacionados ao ambiente (Furlan et al., 2016). Tratando-se dos anuros, variáveis como pluviosidade e temperatura são determinantes para sua organização no espaço, pois, definem diferenciações no período de atividade de forma sazonal (Paula et al., 2015). Ademais, o compartilhamento espacial envolve a utilização de microhabitats diferentes, estando intimamente relacionado aos modos reprodutivos (Duellman \& Trueb, 1986).

A partição de recurso, resultante da redução de possibilidades de competição interespecífica (Pombal Jr, 2010), é uma área de estudo da ecologia necessária para analisar o limite da coexistência entre espécies (Gordon, 2000). Portanto, abordagens sobre partição de recursos entre espécies filogeneticamente relacionadas são importantes, para o melhor entendimento dos fatores que direcionam a organização desses organismos no ambiente (Pombal Jr, 2010). Apenas nas últimas décadas um maior número de estudos fora desenvolvido em prol da obtenção de conhecimento sobre organização e estrutura das comunidades de anfíbios no Brasil (Crema et al., 2014; Gambale et al., 2014; Lipinski \& Santos, 2014).

A maior parte dos estudos sobre partição de recursos por anfíbios foram desenvolvidos para a região da Mata Atlântica (Ferreira et al., 2012), evidenciando uma segregação dos organismos entre ambientes secos e úmidos, além de microambientes específicos para cada padrão reprodutivo. Entretanto, para regiões áridas, considerando a heterogeneidade da paisagem, pesquisas indicam uma maior preferência de espécies por áreas abertas, pois apresentam maior disponibilidade de ambientes para reprodução (Oda et al., 2009; Melo et al., 2013).

Entre os grandes biomas brasileiros é evidente que a Caatinga ainda é o menos conhecido com ênfase na sua biodiversidade (Lewinsohn \& Prado, 2006; Vieira et al., 2007). Por este motivo, pesquisas que visam aspectos ecológicos dos organismos que utilizam esse ambiente, assim como a importância desse bioma são necessárias para o planejamento e tomada de decisões a respeito de sua conservação e restauração. Desta forma, o presente trabalho objetiva descrever o padrão de distribuição espacial e temporal de anuros intimamente relacionados filogeneticamente, usando como modelo espécies da família Leptodactylidae em área com vegetação predominante do bioma Caatinga, no Município de Tabira, Pernambuco, Brasil.

\section{Metodologia}

\section{1 Área de estudo}

O estudo foi realizado no sitio Cachoeira de Maria Mendes (7.507982S/37.505444W) (Figura 1), Município de Tabira, a área está inserida na microrregião do Pajeú, localizada no centro-norte do estado de Pernambuco, próximo ao limite com o estado da Paraíba, inserida na mesorregião do sertão de Pernambuco (Versyple et al., 2015). A região integra o bioma Caatinga, possuindo clima semiárido, em que apresenta altos níveis de radiação solar, em geral a temperatura varia de $17{ }^{\circ} \mathrm{C}$ a $36{ }^{\circ} \mathrm{C}$, possui precipitações irregulares e umidade relativa do ar baixa, com uma altitude de 674 metros (Versyple et al., 2015). 
Figura 1. Localização geográfica do sitio Cachoeira de Maria Mendes, Município de Tabira, na região Nordeste, Estado de Pernambuco, Brasil.

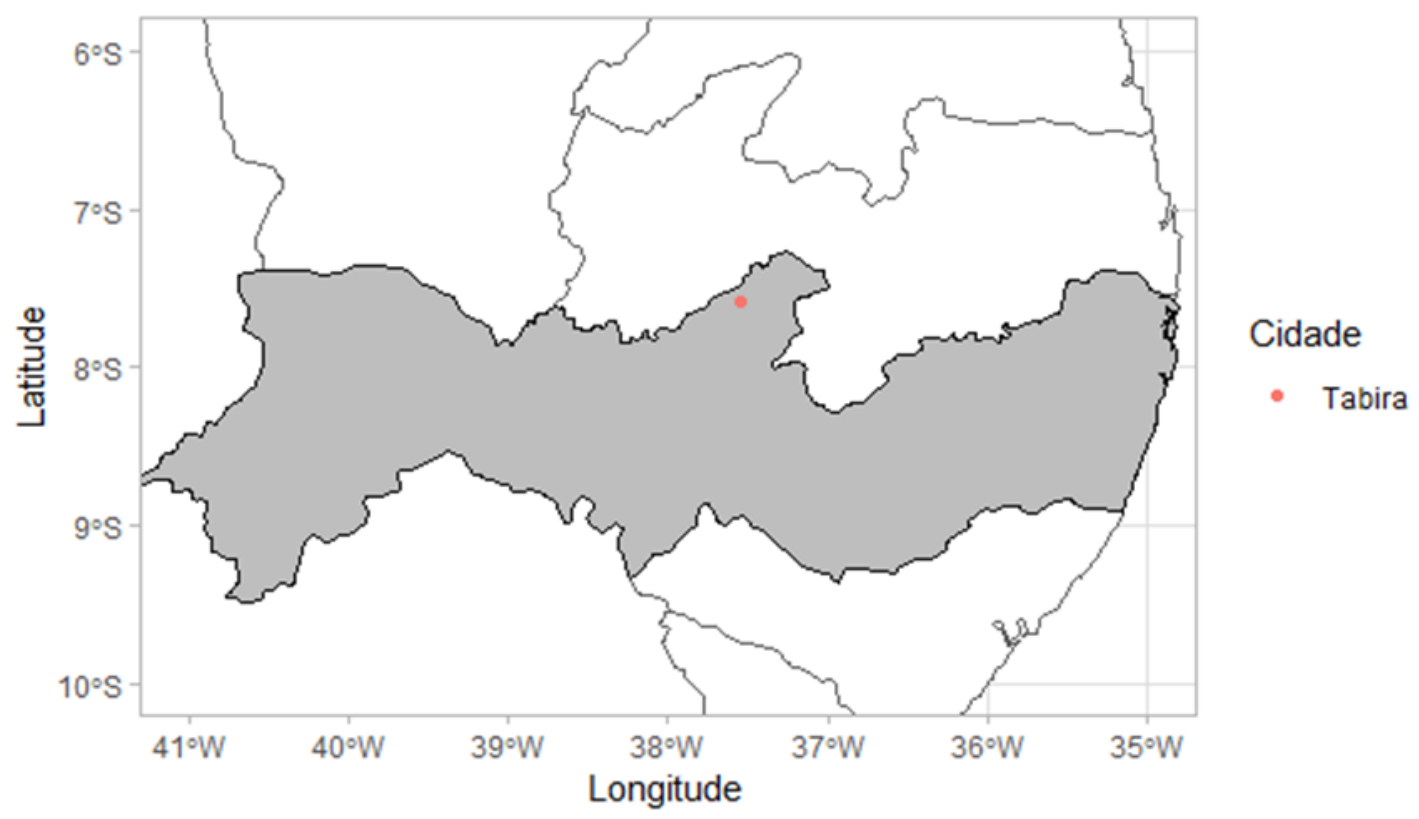

Fonte: Autores.

O estudo foi realizado entre os meses de fevereiro de 2017 e julho de 2018, consistindo em 2 visitas mensais a campo. Foram acompanhadas uma estação seca no ano de 2017 e duas estações chuvosas nos respectivos anos 2017 e 2018. As fitofisionomias foram selecionadas de acordo com o grau de cobertura vegetal, proximidade de corpos d'água e grau de antropização, no qual foram determinados 2 transectos com a utilização de um GPS da marca Garmin (modelo etrex10), sendo o transecto A1 de 500 metros e o A2 de 300 metros, abrangendo a margem de um riacho, e também, um açude presente no local.

\subsection{Procedimento de campo}

A amostragem consistiu em buscas ativas e acústicas no período noturno (das 19:00 às 00:00 horas) com três observadores para cada transecto, totalizando um esforço amostral de 408 horas. Foi utilizado o método de Procura Visual Limitada por Tempo (PVLT) (Campbell \& Christiman, 1982), amostrando todos os possíveis microhabitats das espécies. Foram aferidas as temperaturas dos ambientes, umidade do ar e a condição do tempo para cada avistamento durante o percurso de observação, usando um termohigrômetro digital (modelo kt-908). Foram obtidas as seguintes informações no momento dos avistamentos dos espécimes: (I) horário de avistamento, (II) espécie com base na identificação visual e vocalização, (III) atividade, (IV) habitat, (V) microhabitat.

\subsection{Análises estatísticas}

Todas as análises foram feitas através do Software R nas versões 3.6.3 e 4.1.0 e no Software RStudio. A sobreposição do uso do habitat foi calculada através da versão da equação de MacArthur \& Levins, extraída de Pianka (1973) utilizando os pacotes "spaa"; "xlsx" e "rJava" com 999 aleatorizações. A estatística descritiva foi feita utilizando o programa Microsoft Excel 2016 e representou a riqueza de espécies versus fatores abióticos que poderiam influenciar sua ocorrência. Uma Análise de Componentes Principais (PCA) foi construída com os habitats para evidenciar os sítios em que os Leptodactilídeos possuem maior frequência, os pacotes utilizados foram "vegan" e "factoextra". A relação entre riqueza versus temperatura, umidade e pluviosidade foram estatisticamente testadas usando o Teste Tukey e uma ANOVA. Uma regressão entre as espécies e as 
variáveis ambientais e quais variáveis estão correlacionadas com as espécies. Os valores seguiram grau de significância de $\mathrm{p}<$ 0,05. Neste caso, embora as chuvas possam ocorrer nos meses considerados como secos foi utilizado o critério já adotado na literatura para esta região.

\section{Resultados}

Foi registrado um total de 594 indivíduos, pertencentes a 9 espécies de anfíbios anuros distribuídos na família Leptodactylidae. Leptodactylus troglodytes $\mathrm{n}=(184)$ foi a espécie mais abundante em relação as demais com ocorrência em todos os habitats amostrados.

No que se refere a sobreposição de nicho, a maioria das espécies apresentou sobreposição máxima. A menor sobreposição foi observada entre L. troglodytes e L. fuscus (Tabela 1).

Tabela 1. Valores para sobreposição de nicho espacial (habitat) das espécies encontradas no sitio Cachoeira de Maria Mendes, $(\mathrm{n} \geq 9)$ entre os meses de fevereiro/2017 à junho/2018. Os acrônimos na linha superior e na primeira coluna à esquerda da tabela representam as iniciais dos nomes das espécies. L. vas=Leptodactylus vastus; L. trog=Leptodactylus troglodytes; P. dipl= Pleurodema diplolister $;$ P. cuv $=$ Physalaemus cuvieri $;$ P. alb= Physalaemus albifrons; P. cic $=$ Physalaemus cicada $;$ L. chac $=$ Leptodactylus chaquensis; L. fus= Leptodactylus fuscus e P. poc= Pseudopaludicola pocoto.

\begin{tabular}{|c|c|c|c|c|c|c|c|c|c|}
\hline & L. vas & L. trog & P. dipl & P. cuv & P. alb & P. cic & L. chac & L. fus & P. poc \\
\hline L. vas & - & 0.816 & 1 & 0.75 & 1 & 0.75 & 1 & 0.577 & 0.75 \\
\hline L. trog & & - & 0.816 & 0.816 & 0.816 & 0.612 & 0.816 & 0.471 & 0.816 \\
\hline P. dipl & & & - & 0.75 & 1 & 0.75 & 1 & 0.577 & 0.75 \\
\hline P. cuv & & & & - & 0.75 & 0.75 & 0.75 & 0.577 & 0.5 \\
\hline P. alb & & & & & - & 0.75 & 1 & 0.577 & 0.75 \\
\hline P. cic & & & & & & - & 0.75 & 0.866 & 0.5 \\
\hline L. chac & & & & & & & - & 0.577 & 0.75 \\
\hline L. fus & & & & & & & & - & 0.577 \\
\hline P. poc & & & & & & & & & - \\
\hline
\end{tabular}

Fonte: Autores.

A menor abundância de indivíduos foi observada entre setembro e outubro, a temperatura se manteve constante ao longo dos meses (Figura 2). A pluviosidade oscilou e a abundancia se manteve relativamente constante. Todas as espécies encontradas na área de estudo vocalizaram preferencialmente no período noturno, sendo que ocasionalmente a espécie $P$. pocoto foi avistada em atividade vocal no final da tarde. 
Figura 2. Riqueza de espécies (barra branca), média mensal da temperatura do ar (linha cinza) e média mensal da pluviosidade (linha preta) no período de fevereiro/2017 à junho/2018 na Cachoeira de Maria Mendes, Município de Tabira, Pernambuco, Brasil.

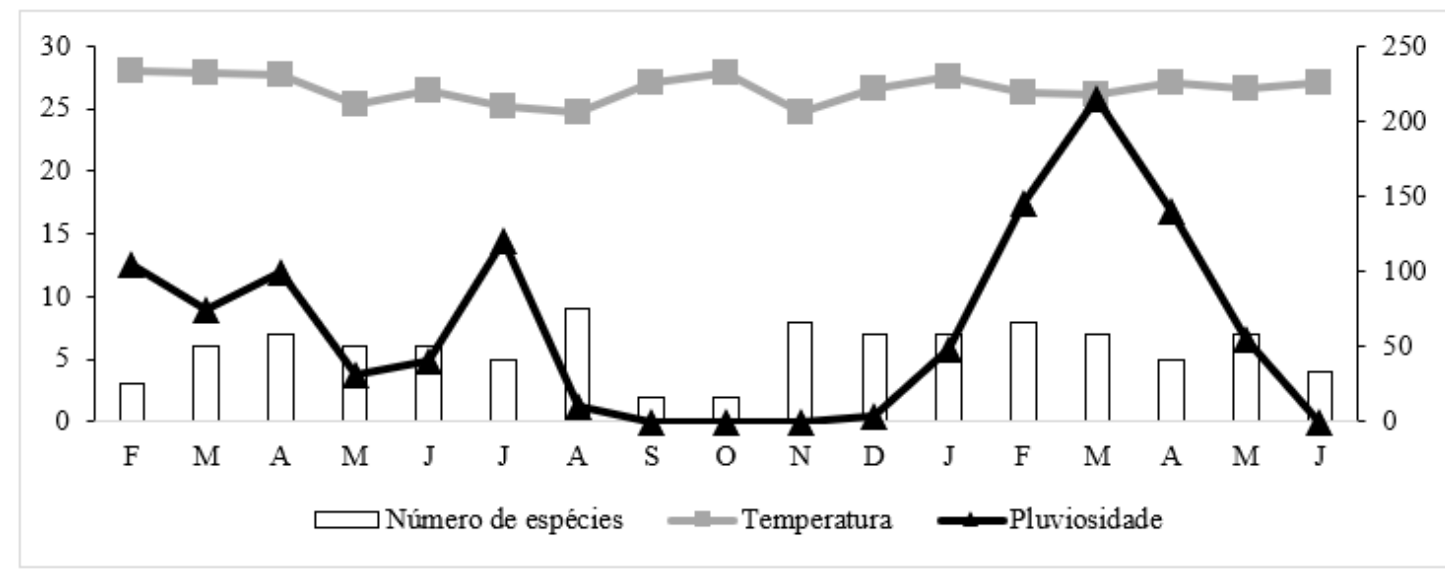

Fonte: Autores.

Os indivíduos da família Leptodactylidae apresentaram constância por ambientes secos e úmidos como os habitats Solo e Açude, respondendo juntos por 55,2\% das demais variáveis.

Figura 3. Biplot Dim1 x Dim2 sobre as variáveis (Groups - Sítios) em áreas de ocorrência relacionadas a cada espécie na Cachoeira de Maria Mendes, Município de Tabira, Pernambuco, Brasil.

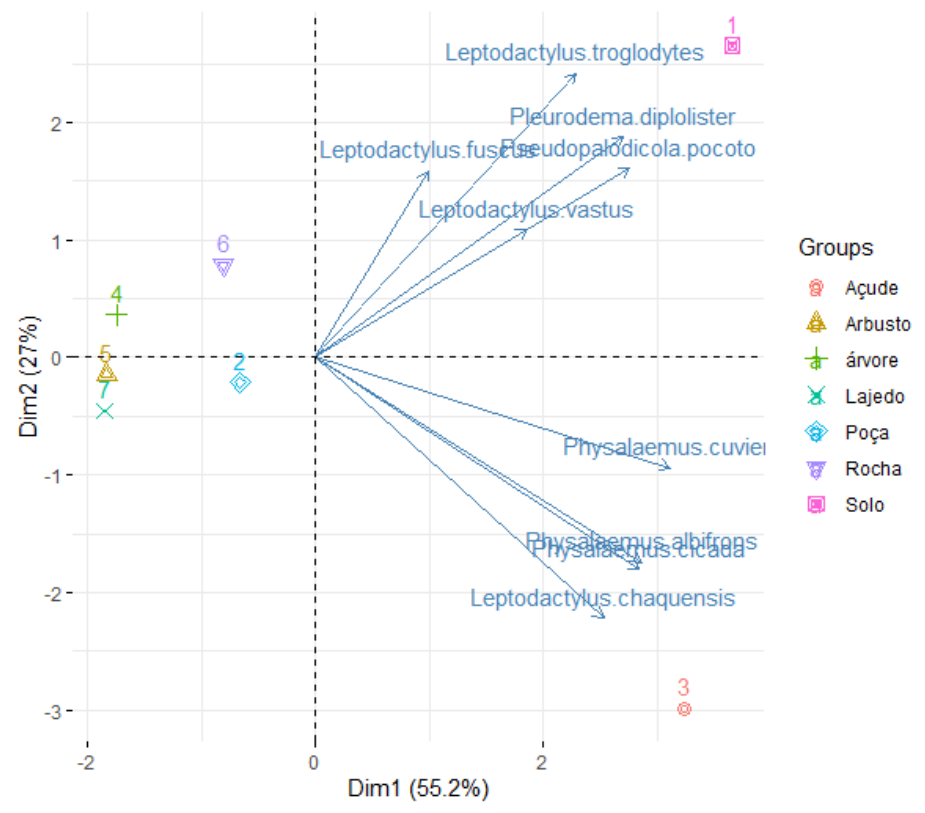

Fonte: Autores.

Foi constatado que houve correlação significativa entre a riqueza de espécies e a pluviosidade, mas não houve correlação entre riqueza de espécies e temperatura. Já a abundância foi significativa tanto com a temperatura quanto com a pluviosidade (Tabela 2), porém, o teste de regressão não apontou significância nas relações entre: Riqueza x Temperatura. 
Tabela 2. Valores obtidos entre Riqueza e Abundância versus fatores abióticos dos leptodactilídeos da Cachoeira de Maria Mendes, Município de Tabira, Pernambuco, Brasil, entre fevereiro/2017 e junho/2018.

\begin{tabular}{llllll}
\hline & Fatores abióticos & ANOVA & \multicolumn{3}{c}{ Regressão } \\
\hline Riqueza & Pluviosidade & $\mathrm{F}=13.93$ & $\mathrm{p}=0.0007$ & $\mathrm{~T}=5.277$ & $\mathrm{p}=0.01$ \\
& Temperatura & $\mathrm{F}=1339$ & $\mathrm{p}=1.1$ & $\mathrm{~T}=51.74$ & $\mathrm{p}=9.6$ \\
Abundância & Pluviosidade & $\mathrm{F}=2.702$ & $\mathrm{p}=0.11$ & $\mathrm{~T}=2.324$ & $\mathrm{p}=0.11$ \\
& Temperatura & $\mathrm{F}=1.98$ & $\mathrm{p}=0.16$ & $\mathrm{~T}=1.99$ & $\mathrm{p}=0.16$ \\
\hline
\end{tabular}

Fonte: Autores.

\section{Discussão}

Os dados indicam que espécies filogeneticamente próximas como L. troglodytes versus L. fuscus obtiveram o menor índice de sobreposição do nicho espacial, de modo que as duas espécies estão ocorrendo no fragmento de mata e em áreas abertas. Diante disso, a teoria contemporânea da coexistência afirma que a manutenção dos organismos em um ambiente está relacionada com a convivência estável por meio da aptidão das espécies ou pela estabilização das interações inter e intraespecíficas por meio de mecanismos como particionamento de recurso (Hillerislambers et al., 2012), portanto, os resultados aqui evidenciados sugerem L. troglodytes e L. fuscus estão mantendo suas populações estáveis, de modo que as espécies utilizam de maneira diferente o nicho espacial.

Estudos com anuros que ocorrem em ambientes áridos mostram que a disponibilidade de água desempenha um papel fundamental na estruturação das assembleias (Protázio et al., 2015; Vieira et al., 2007), sendo esse um provável recurso limitante que estrutura os leptodactilídeos da Cachoeira de Maria Mendes, considerando que o teste de Tukey e a Análise de Variância (ANOVA), demonstraram que a riqueza e abundância é influenciada positivamente pela pluviosidade, portanto, a variável pluviosidade mostra-se determinante para estruturação da população de leptodactilídeos considerando a pequena área amostrada.

Em escala temporal o mês que apresentou maior riqueza de espécies foi agosto de 2017, logo após um pico pluviométrico que ocorreu no mês de julho registrando uma precipitação de $120 \mathrm{~mm}^{3}$. Pesquisas afirmam que modificações de habitats exercem fortes pressões seletivas em espécies especialistas, no entanto, favorecem espécies generalistas quando resulta no surgimento de novas áreas para reprodução (Grandinetti \& Jacobi, 2005), como exemplo os ambientes lênticos, os quais apresentam predominância de leptodactilídeos (Grandinetti \& Jacobi, 2005; De-Carvalho et al., 2008; Pazinato et al., 2011), logo, tais afirmações corroboram com os resultados aqui observados.

De acordo com a Análise de Componentes Principais (PCA) as espécies estão fortemente associadas a ambientes abertos e antropizados, utilizando preferencialmente o solo e açude; já os habitats florestais apresentaram valores inferiores, sendo registradas menores ocorrências em árvores, logo, solo e açude explicaram 55,2\% dos habitats utilizados em comparação aos demais componentes considerados, evidenciando uma alta plasticidade. Deste modo, os dados obtidos corroboram com outros trabalhos realizados na Caatinga (Vieira et al., 2007; Costa et al., 2016).

\section{Conclusão}

Conclui-se, portanto, que os resultados aqui apresentados confirmam pesquisas as quais afirmam que a família Leptodactylidae inclui espécies generalistas que respondem bem a diversos ambientes possuindo hábitos terrestres e semiaquáticos. Ademais, foi constatado que L. troglodytes versus L. fuscus demonstraram o menor valor da sobreposição no uso do habitat em comparação as demais espécies, sendo este um dado importante sobre os aspectos evolutivos da família Leptodactylidae.

Com base nas evidencias aqui abordadas, sugerimos que mais estudos descritivos sejam desenvolvidos para elucidar quais estratégias as espécies filogeneticamente próximas estão utilizando para diminuir o grau de competição interespecífica e 
quais fatores ecológicos estão relacionados na estruturação dessas populações.

\section{Referências}

Ferreira, R. B., Dantas, R. B., \& Tonini, J. F. R. (2012). Distribuição espacial e sazonal de anfíbios em quatro poças na região serrana do Espírito Santo, sudeste do Brasil: influência de corredores florestais. Iheringia, 102(2), 163-169.

De-Carvalho, C. B., De-Freitas, E. B., Faria, R. G. Batista, R. C., Batista, C. C., Coelho, W. A.; \& Bocchiglieri, A. (2008). História natural de Leptodactylus mystacinus e Leptodactylus fuscus (Anura: Leptodactylidae) no Cerrado do Brasil Central. Biota Neotropica, 8(3), $105-115$.

Gordon, C. E. (2000). The coexistence of species. Revista Chilena de Historia Natural, 73, 175-198.

Campbell, H. W., Christiman, S. P. (1982). Field techniques for herpetofaunal community analysis. Wildlife Research Report, 13, $193-200$.

Costa, D. F. S., Oliveira, J. C. D., Oliveira, J. F., Chaves, M. F., Silva, J. N., \& Sousa, T. P. (2016). Dieta de Leptodactylus macrosternum (Amphibia; Anura; Leptodactylidae) no Sertão da Paraíba, Brasil. Revista Verde de Agroecologia e Desenvolvimento Sustentável, 11(4), 123.

Crema, A., Sousa, F. A. C. F., Patelli, L. F. P., Carvalho, R. M. V., \& Mesquita, D. O. (2014). Diversidade e distribuição de anfíbios anuros em matas de galeria do Distrito Federal, Brasil. Revista Nordestina de Biologia, 23(1), 3-27.

Protázio, A. S., Albuquerque, R. L., Falkenberg, L. M., \& Mesquita, D. O. (2015). Acoustic ecology of an anuran assemblage in the arid Caatinga of northeastern Brazil. Journal of Natural History, 49(16), 957-976.

Duellman, W. E., \& Trueb, L. (1986). Biology of amphibians. JHU press.

Furlan, S. A., Lima, E. R. V., Sousa, B. I., \& Sousa, R. M. (2016). Biogeografia: reflexões sobre temas e conceitos. Revista da Anpege, 12 (18), 97-115.

Gambale, P. G., Woitovicz-Cardoso, M., Vieira, R. R., Batista, V. G., Ramos, J., \& Bastos, R. P. (2014). Composição e riqueza de anfíbios anuros em remanescentes de Cerrado do Brasil Central. Iheringia, 104(1), 50-58.

Grandinetti, L., \& Jacobi, C. M. (2005) Distribuição estacional e espacial de uma taxocenose de anuros (Amphibia) em uma área antropizada em Rio AcimaMG. Lundiana, 6(1), 21-28.

Hillerislambers, J., Adler. P. B., Harpoli, W. S., Levini, J. M., \& Mayfild, M. M. (2012). Rethinking community assembly through the lens of coexistence theory. Annual Review of Ecology, Evolution, and Systematics, 43, 227-248.

Versyple, N. I., Machado, J., Andrade, J. S. C. O., \& Wanderley, R. A. (2015). Microrregião Pajeú: economia, clima e desenvolvimento da agricultura através de modelo digital do terreno. Geama, 1(1), 16-30.

Pombal Jr, J. (2010). O espaço acústico em uma taxocenose de anuros (Amphibia) do sudeste do Brasil. Arquivos do Museu Nacional, 68(2), $135-144$.

Lewinsohn, T. M., \& Prado, P. I. (2006). Síntese do Conhecimento Atual da Biodiversidade Brasileira. Avaliação do estado do conhecimento da biodiversidade brasileira, 1, 21-109.

Lipinski, V. M., \& Santos, T. G. (2014). Estrutura e organização espacial de duas comunidades de anuros do bioma pampa. Iheringia, 104(4), 462-469.

Melo, M., Fava, F., Pinto, H. B. A., Bastos, R. P., \& Nomura, F. (2013). Diversidade de Anuros (Amphibia) na reserva extrativista Lago do Cedro e seu entorno, Aruanã, Goiás. Biota Neotropica, 13(2), 205-217.

Oda, F. H., Bastos, R. B., \& Lima, M. A. C. S. (2009). Taxocenose de anfíbios anuros no Cerrado do Alto Tocantins, Niquelândia, Estado de Goiás: diversidade, distribuição local e sazonalidade. Biota Neotropica, 9(4), 219-232.

Pazinato, D. M. M., Oliveira, S. V., Capellari, L. \& Trindade, A. O. (2011). Dieta de Leptodactylus latrans (Steffen, 1815) na Serra do Sudeste, Rio Grande do Sul, Brasil. Biotemas, 24(4), 147-151.

Pianka E. R. (1973). The Structure of Lizard Communities. Annual Review of Ecology and Systematics, 4(1), 53-74.

Vieira, W. L. S., Azarbe, C., \& Santana, G. G. (2007). Composição e distribuição espaço-temporal de anuros no Cariri Paraibano, Nordeste do Brasil. Oecologia brasiliensis, 11(3), 383-396. 